\title{
BMJ Open A cluster-randomised controlled trial of a physical activity and nutrition programme in retirement villages: a study protocol
}

\author{
Anne-Marie Holt, ${ }^{1}$ Jonine Jancey, ${ }^{1,2}$ Andy $\mathrm{H}$ Lee, ${ }^{2}$ Deborah A Kerr, ${ }^{2}$ \\ Andrew P Hills, ${ }^{3}$ Annie S Anderson, ${ }^{4}$ Peter A Howat ${ }^{1,5}$
}

To cite: Holt A-M, Jancey J, Lee $\mathrm{AH}$, et al. A clusterrandomised controlled trial of a physical activity and nutrition programme in retirement villages: a study protocol. BMJ Open 2014;4 e005107. doi:10.1136/ bmjopen-2014-005107

- Prepublication history for this paper is available online. To view these files please visit the journal online (http://dx.doi.org/10.1136/ bmjopen-2014-005107)

Received 21 February 2014 Revised 6 August 2014 Accepted 8 August 2014

\section{CrossMark}

For numbered affiliations see end of article.

Correspondence to Annie-Marie Holt; a.holt@ curtin.edu.au

\section{ABSTRACT}

Introduction: Physical activity levels of Australia's ageing population are declining and coincidentally rates of overweight and obesity are increasing. Adequate levels of physical activity and a healthy diet are recognised as important lifestyle factors for the maintenance of a healthy weight and prevention of chronic diseases. Retirement village (RV) residents rarely engage in physical activity and nutrition programmes offered, with poor attendance and low use of existing facilities such as on-site fitness centres and classes and nutrition seminars. The RV provides a unique setting to access and engage with this older target group, to test the effectiveness of strategies to increase levels of physical activity, improve nutrition and maintain a healthy weight.

Method and analysis: This cluster-randomised controlled trial will evaluate a physical activity, nutrition and healthy weight management intervention for insufficiently active ('not achieving 150 min of moderate-intensity physical activity per week') adults aged 60-75 residing in RV's. A total of 400 participants will be recruited from 20 randomly selected RV's in Perth, Western Australia. Villages will be assigned to either the intervention group $(n=10)$ or the control group $(n=10)$ each containing 200 participants. The Retirement Village Physical Activity and Nutrition for Seniors (RVPANS) programme is a home-based physical activity and nutrition programme that includes educational resources, along with facilitators who will motivate and guide the participants during the 6-month intervention. Descriptive statistics and mixed regression models will be performed to assess the intervention effects. This trial will evaluate an intervention for the modification of health risk factors in the RV setting. Such research conducted in RV's has been limited.

Ethics and dissemination: Curtin University Human Research Ethics Committee (approval number: HR128/2012). Dissemination of the study results will occur through publications, reports, conference presentations and community seminars.

Trial registration number: Australia and New Zealand Clinical Trial Registry (ACTRN12612001168842)
Strengths and limitations of this study

- Use of a theory-based approach to develop a semitailored physical activity, diet and obesity management programme for retirement village residents.

- Challenges in recruiting participants and sustaining them in the intervention programme.

- This is one of the few physical activity and nutriton trials to be conducted in retirement villages settings. This trial will add to our understanding of health behaviours of retirement village residents.

\section{INTRODUCTION}

Australia has an ageing population of which an estimated $25 \%$ will be over the age of 65 by $2042 .{ }^{12}$ Coinciding with increasing age is decreasing physical activity levels and increasing overweight/obesity. In excess of $46 \%$ of those aged 65 and over are insufficiently active and $60 \%$ of older adults are classified as obese or overweight. ${ }^{3}{ }^{4}$ The obesity epidemic represents a significant public health issue, translating into an estimated annual cost of $\$ 21$ billion in Australia, ${ }^{5}$ further highlighting the need for physical activity and nutrition programmes capable of engaging this older group.

In 2009, 5\% of Australians aged 65 and over lived in retirement villages; this figure is expected to rise to $6 \%$ by 2016 and $7.2 \%$ by $2025 .{ }^{6}$ As a consequence, demand for retirement villages as a residential option will increase. $^{7}$ Retirement villages in this study are defined as congregate housing communities that cater for adults aged 55 years and over with independent living units, recreational facilities and social and physical supports allowing residents to age in place while retaining functional independence. ${ }^{8}$ Services offered by retirement villages are important to attract residents with a diverse range of 
needs, especially support for physical activity and healthy eating. ${ }^{9}$

Adults living in retirement villages are typically sedentary with low attendance at offered physical activity programmes. ${ }^{10}$ However, physical activity contributes to independent living, which is a core value of retirement village communities. ${ }^{6}{ }^{9}$ Most physical activity programmes offered in retirement villages are often limited, being predominantly therapy-based (eg, falls prevention) or light recreational activities such as lawn bowls. ${ }^{11}$ Typically, such programmes lack a nutrition component ${ }^{12}$ even though older adults are at high risk of poor nutrition (malnourished) or inadequate nutrition (obesity and overweight). ${ }^{12}$ Evaluation of physical activity and nutrition programmes has been reported in aged care rather than retirement village settings, ${ }^{13}$ while research in the latter has been mainly limited to falls prevention, functional decline, group exercise classes and weight training. ${ }^{14-18}$ In addition, such evaluations have been conducted with small samples, or the programmes are outdated. ${ }^{14}$ There is an urgent need for the development of a physical activity and nutrition intervention for older adults living in retirement villages, which includes rigorous evaluation.

Reshaping physical activity, diet and overweight and obesity is about supporting behaviour change for positive health outcomes. Supporting behaviour change is most effective when interventions also target the social and physical environment, and policy within organisations. ${ }^{19-21}$ Home-based health promotion interventions for community-living people aged 60 and over have been shown to be effective, ${ }^{22} 23$ and an evaluation of a recent physical activity and nutrition programme yielded promising results, indicating potential for such an approach to be adapted to other settings. ${ }^{23}$

This proposed large scale cluster-randomised trial will evaluate a physical activity, nutrition and healthy weight management intervention for adults aged 60-75 years residing in retirement villages, which will ultimately reduce the risk of chronic disease (eg, diabetes, cardiovascular disease and some cancers). ${ }^{23}$ We hypothesise that by the end of the intervention there will be significant improvements $(\mathrm{p}<0.05)$ in physical activity (time/ intensity), dietary behaviours (reduction in dietary fat; increased intakes of fibre, fruit and vegetables) and body composition of the intervention group participants, compared with the control group participants. The Retirement Village Physical Activity and Nutrition for Seniors (RVPANS) study is developed based on a previously validated study whereby the intervention programme and instruments were successfully implemented and tested (pre and post) in older Australian adults. ${ }^{22-24}$

\section{METHODS}

Study design

This cluster-randomised controlled trial will evaluate a physical activity, nutrition and healthy weight management intervention for adults aged 60 to 75 residing in retirement villages in metropolitan Perth, the capital of Western Australia. Data will be collected at two time points-baseline/preintervention and postintervention; see figure 1 . The programme is overseen by the management committee who are independent of the funding body.

\section{Theoretical framework}

This intervention is designed based on SelfDetermination Theory (SDT), Social Cognitive Theory (SCT) and informed by Motivational Interviewing (MI) ${ }^{25-28}$ SDT, SCT and MI can be utilised to construct advice in an autonomous supportive manner to elicit behaviour change. ${ }^{28}{ }^{29}$ Mapping of strategies informed by theory are summarised in table 1 . The theory is intended to explain as well as change behaviour. We will provide feedback on how the participants' behaviours compare with recommendations (eg, fruit and vegetable intake and levels of physical activity during the MI telephone calls). Research indicates that the synergistic effects of working on two or more health-related behaviours at a time may reduce costs and increase effectiveness of interventions. ${ }^{30}$

\section{Recruitment}

\section{Retirement village selection}

Retirement villages eligible for inclusion will have at least 50 residents aged over 60 years. Initial contact will be made with the management and resident committees of the villages. Among those retirement villages that are willing to participate, 20 villages will be randomly selected. These villages will then be randomly assigned to either the intervention group $(n=10)$ or control group $(n=10)$ using a table of random numbers. An independent person from the research centre will perform the randomisation and assessments, and will be blinded from all aspects of allocation and subsequent intervention. A $15 \mathrm{~km}$ radius surrounding retirement villages will be adhered to, ensuring that those villages eligible to participate will be sufficiently separated to avoid any risk of contamination during the intervention. The study is conducted in Perth metropolitan suburbs and within the Peel region of Western Australia that includes a $200+\mathrm{km}^{2}$ area, which is sufficient to draw a representative sample from.

\section{Retirement village participants}

A total of 400 participants will be recruited from the 20 retirement villages. Participants will be required to be aged 60-75; insufficiently active ('not participating in the recommended $150 \mathrm{~min}$ of moderate-intensity physical activity per week') ${ }^{5}$; on no special diet; not involved in another physical activity programme; but able to participate in a low-stress physical activity programme with minimal risk to overall health and well-being. 
Table 1 Mapping of theory and strategies

\begin{tabular}{|c|c|c|}
\hline $\begin{array}{l}\text { Self determination } \\
\text { theory }\end{array}$ & Action & Method \\
\hline $\begin{array}{l}\text { Perceived benefits/ } \\
\text { barriers }\end{array}$ & $\begin{array}{l}\text { Participants have opportunity to identify the } \\
\text { perceived benefits of participation }\end{array}$ & $\begin{array}{l}\text { Educational content via printed resources (booklet, } \\
\text { exercise chart, newsletter), programme ambassador (PA) } \\
\text { guidance and mentoring }\end{array}$ \\
\hline Cue to action & $\begin{array}{l}\text { Identify plan/actions; external support } \\
\text { (practical and emotional), reminder, } \\
\text { sequenced monitoring }\end{array}$ & $\begin{array}{l}\text { Cues via phone contact (mentoring/role modelling) by } \\
\text { PA, newsletters and practical resources, for example, } \\
\text { activity planner, goal setting tips }\end{array}$ \\
\hline Self-efficacy & $\begin{array}{l}\text { Identify barriers, goal setting, programme } \\
\text { tailored }\end{array}$ & $\begin{array}{l}\text { PA role models; demonstrate and practice exercises; } \\
\text { exercises images; recipes; tips. }\end{array}$ \\
\hline \multirow[t]{4}{*}{$\begin{array}{l}\text { Motivational } \\
\text { interviewing }\end{array}$} & $\begin{array}{l}\text { Increase internal motivation to make } \\
\text { healthier choices }\end{array}$ & $\begin{array}{l}\text { PA trained in MI. MI techniques explained and } \\
\text { opportunity to practice. Resources support }\end{array}$ \\
\hline & $\begin{array}{l}\text { Assist participants to increase intrinsic } \\
\text { motivation for health }\end{array}$ & $\begin{array}{l}\text { PA phone contact-focus on positives. Healthier eating/ } \\
\text { physical activity; provide feedback }\end{array}$ \\
\hline & Use of self-reflection and techniques & $\begin{array}{l}\text { Establish personal reasons and techniques to increase } \\
\text { behaviours and then reflect on them }\end{array}$ \\
\hline & Use push-pull motivating techniques & PA trained in push-pull motivating techniques \\
\hline \multicolumn{3}{|c|}{ Social cognitive theory } \\
\hline Environment & Provide socially supportive environment & $\begin{array}{l}\text { Face-face, phone contact, newsletter. Audit: retirement } \\
\text { village managers encouraged to support policy } \\
\text { development -meetings/feedback }\end{array}$ \\
\hline Situation & $\begin{array}{l}\text { Promote benefits of active ageing/corrected } \\
\text { social norms }\end{array}$ & $\begin{array}{l}\text { Resources, age specific activities, peer-aged mentors; } \\
\text { supportive activities and cues }\end{array}$ \\
\hline $\begin{array}{l}\text { Behavioural } \\
\text { capabilities }\end{array}$ & $\begin{array}{l}\text { Opportunities to problem solve perceived } \\
\text { barriers }\end{array}$ & $\begin{array}{l}\text { PA to support development of strategies to overcome } \\
\text { barriers via contacts and resources }\end{array}$ \\
\hline $\begin{array}{l}\text { Expectations/ } \\
\text { expectancies }\end{array}$ & $\begin{array}{l}\text { Demonstrate benefits of physical activity } \\
\text { and healthy eating }\end{array}$ & $\begin{array}{l}\text { Introductory sessions at programme implementation, } \\
\text { newsletters, phone contact, feedback }\end{array}$ \\
\hline Self-control & Resources for self-monitoring & $\begin{array}{l}\text { Activity planner (in booklet), goal setting tools, fitness/ } \\
\text { nutrition tips, suggested activities }\end{array}$ \\
\hline Observational & Activity demonstration & PA model: demonstrate physical activity programme \\
\hline Reinforcement & Use direct/vicarious reinforcement & On-going and regular contact, support and feedback \\
\hline $\begin{array}{l}\text { Emotional coping } \\
\text { response }\end{array}$ & Discuss problems/barriers & $\begin{array}{l}\text { Phone contact, long-term and short term goal setting } \\
\text { (resources+supported by PA) }\end{array}$ \\
\hline $\begin{array}{l}\text { Reciprocal } \\
\text { determinism }\end{array}$ & Behaviour change strategies & $\begin{array}{l}\text { PA, resources support reflection via goal setting, } \\
\text { mentoring, reinforcement, feedback }\end{array}$ \\
\hline
\end{tabular}

\section{Procedure}

Recruitment will occur through postcard distribution via residents' mailboxes. After the reply paid postcards have been returned, an initial contact will be made by telephone to screen the eligibility of potential participants based on the above inclusion criteria. This procedure has been used effectively to enhance recruitment in previous projects on the same age group. ${ }^{21}{ }^{24}$ For the intervention group, there will be a staggered entry into the intervention programme of about 50 participants per week over a 2-month timeframe. Eligible participants will complete a Physical Activity Readiness Questionnaire $(\mathrm{PARQ})^{31}$ at baseline to eliminate those deemed at high risk. During the same period, the control participants will complete two questionnaires and all measurements (anthropometric and accelerometry) at baseline and post-test as their intervention counterparts to minimise any seasonal effect (figure 1).

\section{Measuring Instruments}

Data will be collected on physical activity levels, nutrition behaviour and anthropometric measures at two time points: preintervention/baseline ( 0 months $)$ and postintervention (6 months; figure 1 ).

\section{Primary outcome measures}

Physical Activity-The International Physical Activity Questionnaire (IPAQ Short, last 7 days), as adapted and validated for the Physical PANS project, ${ }^{32}$ will be used to measure changes in habitual physical activity. This validated and reliable instrument has been widely applied and successfully used in our previous studies involving older Australians. $^{22} 2330$

Accelerometer-ActiGraph ActiTrainer activity monitors $^{33} 34$ will be used to measure daily physical activity

\begin{tabular}{lccc}
\hline Study Group & $\begin{array}{c}\text { 0 month } \\
\text { Baseline }\end{array}$ & Intervention & $\begin{array}{c}\text { 6 month } \\
\text { Post-test }\end{array}$ \\
\hline Intervention $(\mathrm{n}=200)$ & $\mathrm{O}_{1}$ & $\mathrm{X}$ & $\mathrm{O}_{2}$ \\
\hline Control $(\mathrm{n}=200)$ & $\mathrm{O}_{1}$ & $\mathrm{O}_{2}$ \\
\hline $\mathrm{O}=$ observation & $\mathrm{X}=$ Intervention &
\end{tabular}

Figure 1 Study design. 
(incidental and programmed activity). Participants are asked to wear an ActiGraph accelerometer for 7 days at baseline and at 6 months. It will be worn at the left hip throughout waking hours, removed only for showering and swimming. Data collection will be set at 1-minute intervals during the day time. This information will be downloaded by researchers to a computer interface according to the manufacturer's specifications, using the software provided. Minute-by-minute data will be summarised into daily average counts (counts/min/day) and activity durations ( $\mathrm{min} /$ day) in specific intensity levels (inactive/light/moderate/vigorous). The accelerometer enables the assessment of intervention effect and provides an objective measure to validate self-report physical activity data.

Nutrition-The Dietary Questionnaire for Epidemiological Studies V.2 (DQES v2) was developed and validated by the Cancer Council of Victoria. ${ }^{35}$ It is a feasible and low-cost assessment method for habitual dietary intake. In addition to food consumption pattern, the resulting nutritional analysis will provide informative details on energy intake, macronutrients, fibre and other nutrients intake profiles.

\section{Secondary outcome measures}

Body composition/physiology-anthropometric measures (ie, height, weight, waist and hip circumferences) will be recorded at baseline and 6 months, along with blood pressure and demographic characteristics (eg, age, gender, education, marital status and country of birth).

Environment-The Audit of Physical Activity Resources for Seniors (APARS) instrument will be applied to detect changes over time in physical and social environment supports for health-enhancing behaviour in the retirement villages. ${ }^{36}$

\section{Intervention}

Description

The intervention will be based on previous programmes specifically designed for older adults. ${ }^{21} 2430$ The prescriptive physical activity intervention will start at a low level with aerobic and strength/flexibility components. The educational materials will provide support through illustrations and tips on how to perform these exercises safely. The walking component will include programmed and incidental physical activity, while the nutrition component will provide advice on healthy eating by promoting higher consumption of fruit, vegetables and fibre and reduced intake of dietary fat. The resources (programme booklet, exercise chart and newsletter) will provide tips, recipes and menu plans and at the same time encourage goal setting for dietary and physical activity behaviours. Overall, the programme is designed to maximise retention of participants through the establishment of personal goals, monitoring of progress, feedback on progress and social support via programme ambassadors. ${ }^{37} 38$

\section{Programme ambassadors}

Programme ambassadors will be recruited, trained and assigned to each retirement village as a liaison person and to assist in programme delivery. They are volunteers sourced from the research team's extensive networks in the local community who have experience in fitness and senior recreational programmes. Ambassadors will be similar aged peers (aged 60-65) and act as guides to the intervention participants. Research has shown that older adults respond better to peer-related leaders (other older adults) rather than a younger demographic. ${ }^{38}$ They will organise the distribution of intervention resources and questionnaires, as well as being responsible for motivational phone/email contact and subsequent participant feedback based on their training on SDT, SCT and MI. They will be supervised by the project manager and conduct their activities under the direction of an accredited dietitian and human movement specialist.

\section{Programme delivery}

Before commencement of the intervention, the programme ambassadors will meet consented participants at their assigned retirement village. They will initially organise the completion of the medical clearance and the PARQ ${ }^{31}$ The baseline questionnaire will be administered to intervention and control groups. The postdata reassessments will be conducted by researchers who are blinded to the treatment allocation and not involved in the baseline data collection. The first phase of the accelerometer distribution will then occur. The role of the programme ambassadors and processes around the implementation of the intervention will be outlined to participants. A week later the programme resources will be distributed during a second visit, and the accelerometers will be collected. Intervention participants will be provided with information on how to use the resources at this second meeting. This includes demonstrating and explaining the exercises from the booklet. As the intervention is delivered, the Ambassadors will conduct motivational telephone calls to assist with individual tailoring of the programme. ${ }^{27-29}$ Such contact will occur initially at 3 weeks and then at bi-monthly intervals. The phone contact will provide feedback, encouragement and support, and guide the goal setting of participants. ${ }^{38} 39$

\section{Intervention resources}

\section{Programme booklet}

Intervention group participants will receive a booklet designed to educate and enhance their nutrition behaviour and physical activity levels through goal setting. The booklet will be based on the PANS model ${ }^{37}$ and tailored specifically to the retirement village setting. It consists of three sections, with the intention to encourage the establishment of nutrition and physical activity goals in line with national recommendations while suiting each individual's needs. Section 1 introduces the programme 
and provides the most recent guidelines on physical activity and nutrition practice for older adults. Section 2 focuses on physical activity participation, planning and goal setting based on SDT, SCT and MI. Section 3 outlines the Dietary Guidelines for Older Australians ${ }^{40}$ and assists with improving eating habits. The booklet gives fitness and nutrition tips, and serves to guide the participants in reaching their goals while providing the most updated information.

\section{Exercise chart and resistance bands}

The exercise chart supplements the programme booklet as a quick and convenient reference. It is a useful resource for motivation and programme adherence. Each intervention participant will also be provided with a resistance band for strength training. The exercise chart includes instructions and photographs depicting the correct and safe way to perform the resistance band exercises. $^{37} 41$

\section{Programme newsletter}

A bi-monthly newsletter distributed by mail and email reinforces the programme messages and provides a link with the intervention retirement villages. It is developed in accordance to SDT and SCT principles and includes MI strategies. ${ }^{27-29}$ Previous research has shown the value of a 1-2 page newsletter containing supplementary information and update on physical activity and nutrition. ${ }^{23}{ }^{26}$ An accredited dietitian and human movement specialist will oversee the content and presentation. The materials will be pretested with the target group (ie, appropriate to their literacy level). All resources are designed to enhance behaviour change with relevant, easy to understand information regarding physical activity and healthy eating. Collaboration with management and resident's committees will create a supportive social and physical environment at each retirement village. Environmental supports may include signage to encourage stair use, access to opportunities for physical activity and peer support (eg, resident-led group walking activities). ${ }^{42}$

\section{Process evaluation}

Process evaluation will be conducted within 3 weeks of start of the intervention, using a brief questionnaire to evaluate participants' perception of the booklet in terms of readability, ease of understanding, usefulness of advice, suitability and relevance to age group. There will be a section for participants to comment specifically on features they like/dislike about the booklet. This method of process evaluation will similarly be applied to other resources and to assess the programme ambassadors. The process evaluation approach has been demonstrated to be an effective 'incentive' for participants to review the materials carefully, thus increasing their level of engagement in the programme. ${ }^{23}$ Adverse events from activity participation will be recorded by the programme ambassadors as well as through process evaluation during the intervention.

\section{Formative research focus groups}

Focus groups involving three retirement villages will be conducted in two phases. The purpose of the first phase is to clarify enablers and barriers to programme delivery and to review potential intervention strategies. Such information is deemed valuable to modify the intervention procedure and resources, as suggested in previous studies, ${ }^{22-23} 37$ so that the intervention programme can be tailored specifically to suit the retirement village setting. The second phase will then test and refine the intervention resources and modify and fine tune programme delivery.

\section{Exit interviews}

At the conclusion of the intervention, a total of 30 intervention participants will be randomly selected to take part in the exit interviews. Programme completers $(n=15)$ as well as non-completers $(n=15)$ will be invited to undertake a face-to-face interview to gain information regarding their perceptions of the programme, the resources and the programme ambassadors. The noncompleters will be asked the reasons for withdrawing. All qualitative data will be transcribed within 2 weeks of interview completion. At least $10 \%$ of such qualitative data will be randomly selected and reviewed. Data will be collated and presented thematically with direct quotes from participants.

\section{Sample size}

In the IPAQ, participation ( $>10 \mathrm{~min}$ in duration) in various modes of physical activity is initially recorded in binary form (yes, no). We expect the intervention group to exhibit significantly higher prevalence of participation in strength exercise, walking and moderate-intensity activities than the control group at 6 months. The power calculations are based on a logistic mixed regression model with the outcome variable being the prevalence of such physical activity participation, and assuming $70 \%$ complete data across the two assessments due to attrition and non-respondents. In the power analyses, small to medium effect sizes (10-20\% change in prevalence estimates) are expected for conservative estimation adjusting for age and gender only. A sample size of $n=400$ (100 per gender by intervention condition) will provide sufficient power of $80 \%$ to detect such moderate effect sizes at $5 \%$ level of significance.

\section{Statistical analysis}

Descriptive statistics will be used to summarise participants' demographic, health characteristics and the outcome variables. The main objective involves the assessment of intervention effect on changes in physical activity and nutrition behaviours over time. For the hierarchical data (repeated measurements of individuals nested within retirement villages) collected over the 
observational period, multilevel two-part models are appropriate for the semicontinuous variables. In estimating the prevalence of physical activity participation, the logistic mixed regression model accounts for the inherent correlation of the observations via random (retirement village) effects, whereas the generalised $\gamma$ mixed regression part, with suitably defined correlation structure, can provide correct statistical inferences concerning the observed physical activity levels. ${ }^{43}$ Multilevel models will also be used to analyse other primary and secondary outcomes of interest. In addition, intention-to-treat analysis will be performed to assess sensitivity of the results to the expected attrition and withdrawal of participants from the RVPANS study.

\section{CONCLUSION}

Retirement villages are expected to increase in numbers over the coming years to accommodate the burgeoning ageing population. They provide a unique setting to access, engage and test strategies to increase physical activity, to improve nutritional intake and maintain a healthy weight. To date, limited research has been conducted. It is therefore timely to evaluate an intervention targeting the modification of health risk factors in retirement villages.

This innovative research will contribute to a better understanding of health behaviours of older adults in the retirement village setting. It has the potential to provide evidence on barriers and enablers to participation in preventative programmes specific to retirement village residents, particularly on the effectiveness of strategies to improve their dietary intake and physical activity levels. Moreover, the findings will contribute to policy, environmental and infrastructure changes that support the adoption and maintenance of such health-enhancing behaviours.

\section{Author affiliations \\ ${ }^{1}$ Western Australian Centre for Health Promotion, Curtin University, Perth, Western Australia, Australia \\ ${ }^{2}$ School of Public Health, Curtin University, Perth, Western Australia, Australia ${ }^{3}$ Griffith Health Institute, Griffith University, Queensland, Australia \\ ${ }^{4}$ University of Dundee, Aberdeen, Scotland \\ ${ }^{5}$ Centre for Behavioural Research in Cancer Control, Curtin University, Perth, Western Australia, Australia}

Contributors A-MH contributed to the study design and protocol development, is responsible for acquisition of data, analysis and interpretation; also contributed to drafting the article, revising it as well as final approval of version to be published. JJ, AHL, DAK, APH, ASA and PAH contributed to the study design and protocol development, are responsible for data analysis and interpretation; also contributed to drafting the article, revising it critically for important intellectual content as well as final approval of version to be published.

Funding This work is supported by a Western Australian Health Promotion Foundation (PO Box 1284, West Perth, Western Australia 6872) research project grant (grant number: 24021).

Competing interests None.

Patient consent Obtained.
Ethics approval The research protocol has been approved by Curtin University Human Research Ethics Committee (approval no HR128/2012).

Provenance and peer review Not commissioned; externally peer reviewed.

Open Access This is an Open Access article distributed in accordance with the Creative Commons Attribution Non Commercial (CC BY-NC 3.0) license, which permits others to distribute, remix, adapt, build upon this work noncommercially, and license their derivative works on different terms, provided the original work is properly cited and the use is non-commercial. See: http:// creativecommons.org/licenses/by-nc/3.0/

\section{REFERENCES}

1. Australian Government. Australia to 2050: future challenges. Canberra: Australian Government, 2010.

2. Australian Government. Australia's demographic challenges. Canberra: The Treasury, 2011.

3. Stevens J, Katz EG, Huxley RR. Associations between gender, age and waist circumference. Eur J Clin Nutr 2010;64:6-15.

4. Australian Institute of Health and Welfare [AIHW]. Australia's Health Series No. 13. Australia's Health 2012. Canberra: AlHW, 2012.

5. Government of Western Australia. Premier's Physical Activity Taskforce: strategic plan 2007-2011. Perth: Government of Western Australia, 2007.

6. Swanson H. Retirement Living Australia: Healthcare and Retirement Living. 2009.

7. Healy J. The benefits of an ageing population. Discussion paper No. 63. The Australian Institute: Australian National University, 2004.

8. Mehrotran A. The village of the future: babyboomers and retirement living. Perth, WA: Colliers Market Report, 2010.

9. Mihalko SL, Wickley KL, Sharpe BL. Promoting physical activity in independent living communities. Med Sci Sports Exerc 2006;38:112-15.

10. Marshall S, Bauer J, Capra S. Are informal carers and community care workers effective in managing malnutrition in the older community? A systematic review of current evidence. J Nutr Health Aging 2013;17:645-51.

11. Miller E, Buys L. Predicting older Australian's leisuretime physical activity. Activities Adaption Aging 2007;31:13-30.

12. Australian Institute of Health and Welfare [AIHW]. Risk factor monitoring. Canberra: AGPS, 2004.

13. Smith RBK, Bryce A, Dyson C, et al. Well for life: improving nutrition and physical activity for residents of aged care facilities: summary report. Melbourne: National Ageing Research Institute \& Dietitians Australia, Victoria, 2000.

14. Lord SR, Castell S, Corcoran J, et al. The effect of group exercise on physical functioning and falls in frail older people living in retirement villages: a randomised controlled trial. J Am Geriatr Soc 2003;51:1685-92.

15. Pahor M, Blair SN, Espeland M, et al. Effects of a physical activity intervention on measures of physical performance: results of the Lifestyle Interventions and Independence for Elders Pilot (LIFE-P). J Gerontology A Biol Sci Med Sci 2006;61:1157-65.

16. Smitheram K. Promoting physical activity amongst the ageing. An evaluation of the Fit for Action program. Perth: University of Western Australia, 2003.

17. Egan K. Three month trial project: implementation of Living Longer Living Stronger program. Perth, Western Australia: Council on the Ageing, 2010.

18. Hart LE, Haaland DA, Baribeau DA, et al. The relationship between exercise and osteoarthritis in the elderly. Clin J Sport Med 2008;18:508-21.

19. Jeon CY, Lokken RP, Hu FB, et al. Physical activity of moderate intensity and risks of type 2 diabetes: a systematic review. Diabetes Care 2007;3:744-52.

20. Crespo CJ, Palmieri MR, Perdomo RP, et al. The relationship of physical activity and body weight with all-cause mortality: results from the Puerto Rico Heart Health program. Ann Epidemiol 2002;12:543-52.

21. Lee A, Howat P, Jancey J, et al. A home-based physical activity and nutrition intervention program for older adults. JAPA 2008;16:S172.

22. Burke L, Lee $\mathrm{A}$, Jancey $\mathrm{J}$, et al. Physical activity and nutrition behavioural outcomes of a home-based intervention program for seniors. Int J Behav Nutr Phys Act 2013;10:14.

23. Burke L, Jancey J, Howat $P$, et al. Physical activity and nutrition for seniors (PANS): randomized controlled trial. BMC Public Health 2010;10:751. 
24. Burke L, Lee A, Pasalich M, et al. Effects of a physical activity and nutrition program for seniors on body mass index and waist-to-hip ratio: A randomised controlled trial. Prev Med 2012;54:397-401.

25. Vansteenkiste M, Sheldon KM. There's nothing more practical than good theory: integrating motivational interviewing and self-determination theory. Br J Clin Psychol 2006;45(Pt 1):63-82.

26. Lonsdale $\mathrm{C}$, Hall $\mathrm{A}$, Williams $\mathrm{G}$, et al. Communication style and exercise compliance in physiotherapy [CONNECT]. A cluster randomised controlled trial to test a theory -based intervention to increase chronic low back pain patients' adherence to physiotherapists' recommendations: study rationale, design and methods. BMC Musculoskelet Disord 2012;13:104.

27. Resnicow K, McMaster F. Motivational interviewing: moving from why to how with autonomy support. Int J Behav Nutr Phys Act 2012;9:19.

28. Cress ME, Buchner DM, Prochaska T, et al. Best practices for physical activity programs and behavior counseling in older adult populations. J Aging Phys Act 2005;13:61-74.

29. Clark PG, Blissmer BJ, Greene GW, et al. Maintaining exercise and SENIOR project II: study design and methodology. Contemp Clin Trials 2011;32:129-39.

30. Burke L, Jancey J, Howat P, et al. Physical activity and nutrition program for seniors: PANS: a protocol of a randomised controlled trial. BMC Public Health 2010;10:751.

31. Cardinal BJ, Cardinal MK. Preparticipation physical activity screening with a racially diverse, older adults sample: comparison of the original and revised physical activity readiness questionnaires. Res Q Exerc Sport 2000;71:302-7.
32. Grimm EK, Swartz AM, Hart T, et al. Comparison of the IPAQ-short form and accelerometry predictions of physical activity in older adults. J Aging Phys Act 2012;20:64-79.

33. Hart T, Swartz AM, Cashin SE, et al. How many days of monitoring predict physical activity and sedentary behavior in older adults? Int J Behav Nutr Phys Act 2011;8:62-5.

34. David MG, Fox KR. Physical activity patterns assessed by accelerometry in older people. Euro J Appl Physiol 2007;100:581-9.

35. Cancer Council Victoria. Dietary Questionnaire Epidemiological Studies V 2. Victoria, Australia, 2005.

36. Kerr J, Carlson JA, Sallis JF, et al. Assessing health-related resources in senior living residences. J Aging Stud 2011;25:206-14.

37. Burke L, Howat $P$, Jancey $\mathrm{J}$, et al. A nutrition and physical activity booklet to engage seniors. BMC Res Notes 2008;1:1-7.

38. Dorgo S, King GA, Brickey GD. The application of peer mentoring to improve fitness in older adults. J Aging Phys Act 2009;17:344-61.

39. Witham MD, Avenell A. Interventions to achieve long-term weight loss in older people: a systematic review and meta-analysis. Age Ageing 2010;39:176-84.

40. National Health and Medical Research Council [NHMRC]. Dietary guidelines for older people 2012. Canberra: NHMRC, 2012.

41. Van Norman KA. Resistance band exercises improves older adults body strength. Illinois, USA: Human Kinetics, 2012.

42. Holt AM, Howat $\mathrm{P}$, Alfonso $\mathrm{H}$. Evaluation of a recreational team game for seniors: Lifeball—a case study. Healthy Lifestyles J 2009;56:17-21.

43. Lee $\mathrm{AH}$, Zhao $\mathrm{Y}$, Yau $\mathrm{K}$, et al. How to analyse longitudinal multilevel physical activity data with many zeros? Prev Med 2010;51:476-81. 